\title{
Conference Paper \\ On the Issue of Radiation-Induced Instability in Binary Solid Solutions
}

\author{
Santosh Dubey, S. K. Joshi, and B. S. Tewari \\ Department of Physics, University of Petroleum and Energy Studies, Dehradun 248007, India \\ Correspondence should be addressed to Santosh Dubey; santosh.dubey@ddn.upes.ac.in
}

Received 4 February 2014; Accepted 18 March 2014; Published 3 April 2014

Academic Editors: R. K. Shivpuri and G. N. Tiwari

This Conference Paper is based on a presentation given by Santosh Dubey at "National Conference on Advances in Materials Science for Energy Applications" held from 9 January 2014 to 10 January 2014 in Dehradun, India.

Copyright (C) 2014 Santosh Dubey et al. This is an open access article distributed under the Creative Commons Attribution License, which permits unrestricted use, distribution, and reproduction in any medium, provided the original work is properly cited.

The stability of a binary solid solution under irradiation has been studied. This has been done by performing linear stability analysis of a set of nonlinear reaction-diffusion equations under uniform irradiation. Owing to the complexity of the resulting system of eigenvalue equations, a numerical solution has been attempted to calculate the dispersion relations. The set of reaction-diffusion equations represent the coupled dynamics of vacancies, dumbbell-type interstitials, and lattice atoms. For a miscible system (Cu-Au) under uniform irradiation, the initiation and growth of the instability have been studied as a function of various control parameters.

\section{Introduction}

Materials under irradiation are a class of dissipative systems driven far away from the equilibrium state due to production of high densities of defects. These systems decay to lower energy metastable states by the process of phase transformation, microstructure evolution, spatial organization of compositional fluctuations and microstructural elements, and so forth [1]. The spatial organization of compositional fluctuations and microstructural elements happens when the material becomes unstable to infinitesimal perturbations in the order parameter characterizing the pattern: for example, in the case of compositional fluctuations, the order parameter is the composition, whereas, in the case of microstructural elements, the order parameter is the density of these elements. Well known examples of spatial organization of microstructural elements are voids [2-4] and bubble lattices $[5,6]$, precipitate ordering [7], and ordering of vacancy dislocation loops [8]. On the other hand, spatial organization of compositional fluctuations has been observed in two types of material systems. In thermodynamically unstable systems (e.g., Fe-35\%Ni-Cr), the spatial organization of compositional fluctuations takes place when a sluggish long wavelength spinodal-type instability in the composition is accelerated under irradiation, which lead to decomposition of the solid solution; long-term thermal annealing of the irradiated specimen has no effect on the state of the material [9]. On the other hand, in thermodynamically stable systems (e.g., $\mathrm{Fe}-15 \% \mathrm{Ni}-\mathrm{Cr}$ ), the origin of compositional fluctuations is solely due to irradiation. When irradiation is turned off and irradiated sample is annealed for long time, the material attains its homogeneous state [10]. The presence of spatial structures with definite characteristics has strong influence on the physical and mechanical properties of the materials. Therefore, it is important to understand the origin of these structures; the first step towards that goal is to figure out under what conditions the material may become unstable to infinitesimal perturbations with respect to its uniform state.

The issue of solid solution instability under irradiation has been addressed by several researchers in the past. These investigations may be broadly categorized into two major themes. In one class of investigation, the state of the material under irradiation is represented by a coupled system of reaction-diffusion equations in defect and material component fields. In these formulations, the origin and subsequent growth of the instability have been attributed to the presence of vacancy-interstitial recombination reactions, assisted by off-diagonal terms of the diffusion matrix [11-15]. In the other 
class of investigations, first proposed by Martin [16], the state of a material is described by the evolution of one conserved order parameter in the Cahn-Hilliard type framework. In this formulation, the evolution of the order parameter is governed by two competing dynamics: fixed length athermal ballistic exchanges and thermal diffusion. Martin's approach was extended by Enrique and Bellon for arbitrary length ballistic exchanges, in order to construct a phase diagram identifying three possible regimes of material response as a function of forcing parameters: these regions have been identified as solid solution, patterning, and macroscopic phase separation $[17,18]$. The applicability of this model in solid solutions under high temperature irradiation is questionable, because, under high temperature irradiation, the defects are present in nonequilibrium proportions and are sufficiently mobile. Therefore, the response of the material should be governed by a coupled defect (nonconserved) and conserved dynamics, instead of conserved dynamics only.

In the present study, the instability of a solid solution under irradiation has been investigated. For a binary system under irradiation, there are six species in the model: three off-lattice dumbbell interstitials, vacancy, and two material components. All the defect species diffuse either by vacancy mechanism or by interstitialcy mechanism. The diffusion of material components is coupled to the diffusion of the defects. The defects participate in two types of reactions: vacancyinterstitial recombination and change in dumbbell type when a dumbbell interstitial encounters a lattice atom of different type. The defect production has been considered uniform in space and time. The model consists of a set of six coupled nonlinear reaction-diffusion equations with uniform source. For a miscible system $(\mathrm{Cu}-\mathrm{Au})$ under irradiation, the linear stability analysis of the reaction-diffusion equations shows that the solid solution indeed becomes unstable when a set of control parameters (temperature, defect production rate, and initial alloy composition) is varied in a certain way.

Section 2 briefly outlines the summary of the model presented elsewhere [19], followed by the linear stability analysis of the model equations under uniform irradiation in Section 3. The results of the linear stability analysis have been presented in Section 4. Section 5 presents the conclusion of the present work.

\section{Model Summary}

We consider an ideal, concentrated binary solid solution, $A B$, under irradiation. There are six species in the model: three types of dumbbell interstitials ( $\mathrm{AA}, \mathrm{BB}$, and $\mathrm{AB}$ ), vacancy, and $A$ and $B$ lattice atoms. Under operating temperatures of nuclear reactors, the defects are sufficiently mobile. All the defect species diffuse either via vacancy mechanism or via interstitialcy mechanism. The diffusion of the material components is mediated by the defects. In addition to diffusion, defects also participate in two types of reactions: vacancyinterstitial recombination and change in the dumbbell-type reactions. Change in the dumbbell type happens when a dumbbell encounters a lattice atom of different type than its constituents. In this formulation, $\mathrm{A}$ is a faster diffusing species both by vacancy and interstitialcy mechanism, whereas B is the slower diffusing species. Evolution of all the species is governed by reaction-diffusion type dynamics with uniform defect generation term,

$$
\frac{\partial C_{i}}{\partial t}=-\nabla \cdot J_{i}+R_{i}+S_{i},
$$

where $J_{i}=\sum D_{i k} \nabla C_{k}, R_{i}$, and $S_{i}$ are the flux, reaction, and source terms of $i$ th species $(i=\mathrm{AA}, \mathrm{BB}, \mathrm{AB}, \mathrm{V}, \mathrm{A}, \mathrm{B})$, respectively; $D_{i k}$ is an $n \times n$ matrix of concentrationdependent diffusion coefficients, where $n$ is the number of species in the model; $C_{k}$ is the fractional concentration of $k$ th species. Derivation of the defect fluxes has been carried out in the Fickian way. For simplicity in the derivation, the solid solution is considered isotropic and the correlation effects between successive defect jumps have also been ignored. These assumptions led us to derive the flux expressions in 1D and generalize into higher dimensions in a straightforward manner. Although these assumptions may introduce a certain degree of uncertainty in quantitative estimations, they will not affect the nature of the solution we are aiming at. The details of the derivation for $J_{i}$ and $R_{i}$ have been presented somewhere else [19]. For source terms, $S_{i}$, we have used the following expressions:

$$
\begin{gathered}
S_{\mathrm{AA}}=K_{0} C_{\mathrm{A}}^{2}, \\
S_{\mathrm{BB}}=K_{0} C_{\mathrm{B}}^{2}, \\
S_{\mathrm{AB}}=2 K_{0} C_{\mathrm{A}} C_{\mathrm{B}}, \\
S_{v}=S_{\mathrm{AA}}+S_{\mathrm{BB}}+S_{\mathrm{AB}}, \\
S_{\mathrm{A}}=-2 S_{\mathrm{AA}}-S_{\mathrm{AB}}, \\
S_{\mathrm{B}}=-2 S_{\mathrm{BB}}-S_{\mathrm{AB}},
\end{gathered}
$$

such that

$$
S_{\mathrm{AA}}+S_{\mathrm{BB}}+S_{\mathrm{AB}}+S_{v}+S_{\mathrm{A}}+S_{\mathrm{B}}=0,
$$

where $K_{0}$ is defect production rate.

Before performing linear stability analysis, the system of reaction-diffusion equations has been nondimensionalized with respect to the intrinsic length and time scales of the system. This has been achieved by the following transformations:

$$
\begin{gathered}
(x, y, z) \longrightarrow l(\tilde{x}, \tilde{y}, \widetilde{z}) \Longrightarrow \nabla \longrightarrow l^{-d} \widetilde{\nabla}, \\
t \longrightarrow \tau \tilde{t},
\end{gathered}
$$

where $d$ represents the dimension. In the present study, we have taken $d=2$. The time scale $(\tau)$ has been defined as the inverse of the fastest reaction rate, $K_{\max }$, whereas length scale $(l)$ depends upon the fastest diffusing rate, $D_{\max }$,

$$
\tau=\frac{1}{K_{\max }}, \quad l=\sqrt{\frac{D_{\max }}{K_{\max }} .}
$$

The nondimensionalized system of equations may be expressed as

$$
\frac{\partial C i}{\partial \widetilde{t}}=-\widetilde{\nabla} \cdot \widetilde{J} i+\widetilde{R} i+\widetilde{S} i
$$




\section{Linear Stability Analysis}

The concentrations $C_{i}$ in the nondimensionalized system of (6) have been perturbed by an infinitesimal amount, $\delta C_{i}$, about its uniform value $\widehat{C}_{i}$,

$$
\delta C_{i}=C_{i}-\widehat{C}_{i} .
$$

Plugging (7) in (6) and considering quasi-static approximation, that is, when the stability of the uniform state is calculated by considering them stationary at each moment, we get a linearized system of equations in terms of perturbations in the concentrations,

$$
\frac{\partial \delta C i}{\partial t}=-\tilde{\nabla} \cdot \widetilde{J} i(\delta \mathbf{C})+\widetilde{R} i(\delta \mathbf{C})+\widetilde{S} i(\delta \mathbf{C}),
$$

where $\delta \mathbf{C}$ represents a vector of perturbations in the concentration of other species. As according to [20, 21], although quasi-static approximation may not be able to capture the growth rate of the perturbations correctly, it is able to predict the instability threshold quite accurately. In this regard, one will expect instability when at least one of the eigenvalues of the evolution matrix has a positive real part.

The perturbations grow in space and time as follows:

$$
\delta C_{i}=\eta_{i} e^{\omega t+i k x},
$$

where $\eta_{i}$ is the amplitude of the perturbation in the $i$ th species concentration, $\omega$ is the growth factor, and $k$ is the wavevector of the sinusoidal perturbations. Plugging (9) in the linearized system of (8), we get six eigenvalue equations,

$$
\begin{aligned}
\omega \eta_{\mathrm{AA}}= & \xi_{\mathrm{AA}}^{(\mathrm{AA})} \eta_{\mathrm{AA}}+\xi_{\mathrm{AB}}^{(\mathrm{AA})} \eta_{\mathrm{AB}}+\xi_{v}^{(\mathrm{AA})} \eta_{v} \\
& +\xi_{\mathrm{A}}^{\mathrm{AA})} \eta_{\mathrm{A}}+\xi_{\mathrm{B}}^{(\mathrm{AA})} \eta_{\mathrm{B}}, \\
\omega \eta_{\mathrm{BB}}= & \xi_{\mathrm{BB}}^{(\mathrm{BB})} \eta_{\mathrm{BB}}+\xi_{\mathrm{AB}}^{(\mathrm{BB})} \eta_{\mathrm{AB}}+\xi_{v}^{(\mathrm{BB})} \eta_{v} \\
& +\xi_{\mathrm{A}}^{(\mathrm{BB})} \eta_{\mathrm{A}}+\xi_{\mathrm{B}}^{(\mathrm{BB})} \eta_{\mathrm{B}}, \\
\omega \eta_{\mathrm{AB}}= & \xi_{\mathrm{AA}}^{(\mathrm{AB})} \eta_{\mathrm{AA}}+\xi_{\mathrm{BB}}^{(\mathrm{AB})} \eta_{\mathrm{BB}}+\xi_{\mathrm{AB}}^{(\mathrm{AB})} \eta_{\mathrm{AB}} \\
& +\xi_{v}^{(\mathrm{AB})} \eta_{v}+\xi_{\mathrm{A}}^{(\mathrm{AB})} \eta_{\mathrm{A}}+\xi_{\mathrm{B}}^{(\mathrm{AB})} \eta_{\mathrm{B}}, \\
\omega \eta_{v}= & \xi_{\mathrm{AA}}^{(v)} \eta_{\mathrm{AA}}+\xi_{\mathrm{BB}}^{(v)} \eta_{\mathrm{BB}}+\xi_{\mathrm{AB}}^{(v)} \eta_{\mathrm{AB}} \\
& +\xi_{v}^{(v)} \eta_{v}+\xi_{\mathrm{A}}^{(v)} \eta_{\mathrm{A}}+\xi_{\mathrm{B}}^{(v)} \eta_{\mathrm{B}}, \\
\omega \eta_{\mathrm{A}}= & \xi_{\mathrm{AA}}^{(\mathrm{A})} \eta_{\mathrm{AA}}+\xi_{\mathrm{BB}}^{(\mathrm{A})} \eta_{\mathrm{BB}}+\xi_{\mathrm{AB}}^{(\mathrm{A})} \eta_{\mathrm{AB}} \\
& +\xi_{v}^{(\mathrm{A})} \eta_{v}+\xi_{\mathrm{A}}^{(\mathrm{A})} \eta_{\mathrm{A}}+\xi_{\mathrm{B}}^{(\mathrm{A})} \eta_{\mathrm{B}}, \\
\omega \eta_{\mathrm{B}}= & \xi_{\mathrm{AA}}^{(\mathrm{B})} \eta_{\mathrm{AA}}+\xi_{\mathrm{BB}}^{(\mathrm{B})} \eta_{\mathrm{BB}}+\xi_{\mathrm{AB}}^{(\mathrm{B})} \eta_{\mathrm{AB}} \\
& +\xi_{v}^{(\mathrm{B})} \eta_{v}+\xi_{\mathrm{A}}^{(\mathrm{B})} \eta_{\mathrm{A}}+\xi_{\mathrm{B}}^{(\mathrm{B})} \eta_{\mathrm{B}},
\end{aligned}
$$

where $\xi_{i}^{j}(i, j=\mathrm{AA}, \mathrm{BB}, \mathrm{AB}, \mathrm{v}, \mathrm{A}, \mathrm{B})$ are complicated functions of $k$, concentrations, and diffusion and reaction coefficients. Equations (10) may also be expressed in a condense form as

$$
\mathbf{A} \eta=\omega \eta .
$$

Owing to complexity of the analytical estimation of the eigenvalues of the evolution matrix $\mathbf{A}$ (matrix of $\xi$ coefficients) and hence the dispersion relations, a numerical estimation of the eigenvalues has been made using eig function of Matlab.

\section{Results and Discussions}

Considering $\mathrm{Cu}-\mathrm{Au}$ as the system of interest [22], the system of (11) has been solved as an eigenvalue problem to get six eigenvalues $\left(\omega_{1}, \ldots, \omega_{6}\right)$. In this alloy, $\mathrm{Cu}$ is a faster and $\mathrm{Au}$ is a slower diffusing species. Imaginary part of all the eigenvalues is zero, which means that the system's response is nonoscillatory. Also, only one out of six eigenvalues $\left(\omega_{6}\right)$ has been found to change sign from a negative to a positive value under a specific set of control parameters. Other eigenvalues remain always negative. Figure 1 shows the behavior of all the eigenvalues with respect to the wavevector $k$. In this figure, all the stable and unstable eigenvalues have been displayed. We see that, out of six eigenvalues, only one $\left(\omega_{6}\right)$ becomes unstable under the prevailing irradiation conditions and temperature. Other eigenvalues are stable for all $k$ values. This shows that the material under irradiation indeed becomes unstable under a certain set of irradiation conditions and remains stable otherwise.

\section{Conclusion}

Stability of a binary solid solution under irradiation has been studied. There are six species in the model: three dumbbells as off-lattice species, vacancies, and two material components as on-lattice species. The temporal evolution of the solid solution is described by a set of coupled nonlinear reaction-diffusion equations in all the species concentration. Defects diffuse either by vacancy mechanism (vacancies) or by interstitialcy mechanism (dumbbells). The diffusive flux of the defects has been derived in the Fickian framework by considering the solid solution to be isotropic and ideal. The correlation effects in the defect jumps have also been neglected. The flux of the atomic species is coupled to the defect fluxes. Defects also participate in two types of reactions: recombination and change in the dumbbell type. Stability of the solid solution has been investigated by performing linear stability analysis of the set of six reactiondiffusion equations with respect to the uniform state of the binary solid solution under quasi-static approximation [20, 21]. For a miscible $\mathrm{Cu}-\mathrm{Au}$ solid solution, the resulting system of eigenvalue equations has been solved numerically to calculate the dispersion relations showing a relation between the growth factors (eigenvalues) and wavevectors of the sinusoidal perturbations. Real part of one of the growth factors changes sign from a negative to a positive value, signifying the presence of unstable eigenmodes under specific simulation conditions. When simulation conditions are varied beyond the instability threshold values, the growth factor and the window of unstable eigenmodes change. The results obtained in this investigation indicate that, under specific simulation conditions, the binary solid solution indeed becomes unstable to infinitesimal sinusoidal perturbations, which might 

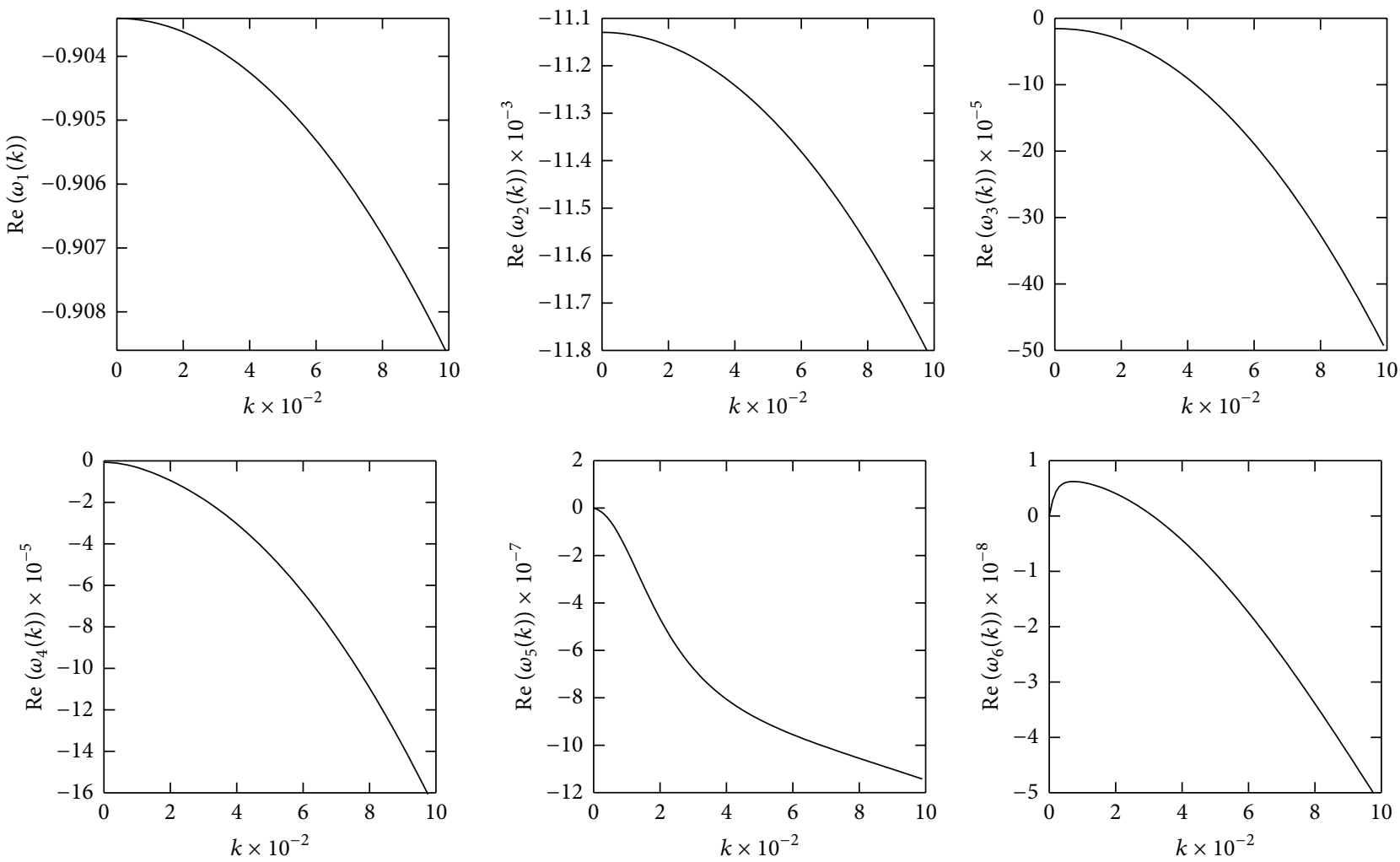

Figure 1: Stable $\left(\omega_{1} \ldots \omega_{5}\right)$ and unstable $\left(\omega_{6}\right)$ growth factors (eigenvalues) of the system calculated at temperature $850 \mathrm{~K}$ and a dpa rate of 2 $\times 10^{-6} \mathrm{dpa} / \widetilde{t}$. The fractional alloy concentration is $C_{\mathrm{Cu}}=0.1$ and $C_{\mathrm{Au}}=0.9$.

result in the formation of stationary self-organized dissipative structures or composition patterns.

\section{Conflict of Interests}

The authors declare that there is no conflict of interests regarding the publication of this paper.

\section{References}

[1] M. C. Cross and P. C. Hohenberg, "Pattern formation outside of equilibrium," Reviews of Modern Physics, vol. 65, no. 3, pp. 851-1112, 1993.

[2] J. H. Evans, "Observations of aregular void array in high purity molybdenum irradiated with $2 \mathrm{MeV}$ nitrogen ions," Nature, vol. 229, no. 5284, pp. 403-404, 1971.

[3] J. Evans, "Observations of a regular void array in high purity molybdenum and T.Z.M. irradiated at high temperatures with $2 \mathrm{MeV}$ nitrogen ions," Radiation Effects, vol. 10, no. 1-2, pp. 55$60,1971$.

[4] K. Krishan, "Kinetics of void-lattice formation in metals," Nature, vol. 287, no. 5781, pp. 420-421, 1980.

[5] S. L. Sass and B. L. Eyre, "Diffraction from void and bubble arrays in irradiated molybdenum," Philosophical Magazine, vol. 27, no. 6, pp. 1447-1453, 1973.

[6] P. B. Johnson, D. J. Mazey, and J. H. Evans, "Bubble structures in $\mathrm{He}^{+}$irradiated metals," Radiation Effects, vol. 78, no. 1-4, pp. 147-156, 1982.
[7] J. H. Evans and D. J. Mazey, "Evidence for solid krypton bubbles in copper, nickel and gold at 293K," Journal of Physics F: Metal Physics, vol. 15, no. 1, article 001, pp. L1-L6, 1985.

[8] W. Jager, P. Ehrhart, and W. Schilling, "Dislocation patterning under irradiation," in Nonlinear Phenomena in Materials Science, G. Martin and L. Kubin, Eds., chapter 2, p. 279, Transtech, Aedermannsdorf, Switzerland, 1988.

[9] F. A. Garner, J. M. McCarthy, K. C. Russell, and J. J. Hoyt, "Spinodal-like decomposition of Fe-35Ni and Fe-Cr-35Ni alloys during irradiation or thermal aging," Journal of Nuclear Materials, vol. 205, pp. 411-425, 1993.

[10] C. M. Shepherd and T. M. Williams, "Intragranular compositional oscillations in neutron-irradiated austenitic steels," Journal of Nuclear Materials, vol. 168, no. 3, pp. 337-339, 1989.

[11] G. Martin, "Contribution of dissipative processes to radiationinduced solid-solution instability," Physical Review B, vol. 21, no. 6, pp. 2122-2130, 1980.

[12] G. Martin, "Long-range periodic decomposition of irradiated solid solutions," Physical Review Letters, vol. 50, no. 4, pp. 250252, 1983.

[13] K. Krishan and C. Abromeit, "Calculation of radiation-induced instability in concentrated alloys," Journal of Physics F: Metal Physics, vol. 14, no. 5, article 008, pp. 1103-1116, 1984.

[14] S. M. Murphy, "Instabilities in concentrated alloys under irradiation," Philosophical Magazine, vol. 58, no. 2, pp. 417-433, 1988.

[15] C. Abromeit and G. Martin, "Dynamical phase changes induced by point defect fluxes under irradiation," Journal of Nuclear Materials, vol. 271-272, pp. 251-255, 1999. 
[16] G. Martin, "Phase stability under irradiation: ballistic effects," Physical Review B, vol. 30, no. 3, pp. 1424-1436, 1984.

[17] R. A. Enrique and P. Bellon, "Compositional patterning in systems driven by competing dynamics of different length scale," Physical Review Letters, vol. 84, no. 13, article 2885, 2000.

[18] R. A. Enrique and P. Bellon, "Compositional patterning in immiscible alloys driven by irradiation," Physical Review B, vol. 64, no. 13, Article ID 134111, 2001.

[19] S. Dubey and A. El-Azab, "Irradiation-induced composition patterns in binary solid solutions," Journal of Applied Physics, vol. 114, no. 12, Article ID 124901, 2013.

[20] N. M. Ghoniem and D. Walgraef, "Evolution dynamics of 3D periodic microstructures in irradiated materials," Modelling and Simulation in Materials Science and Engineering, vol. 1, no. 5, article 001, pp. 569-590, 1993.

[21] B. S. Jhaveri and G. M. Homsy, "The onset of convection in fluid layers heated rapidly in a time-dependent manner," Journal of Fluid Mechanics, vol. 114, pp. 251-260, 1982.

[22] T. Hashimoto, Y. Isobe, and N. Shigenaka, "A model for radiation-induced segregation in fcc binary alloys," Journal of Nuclear Materials, vol. 225, pp. 108-116, 1995. 

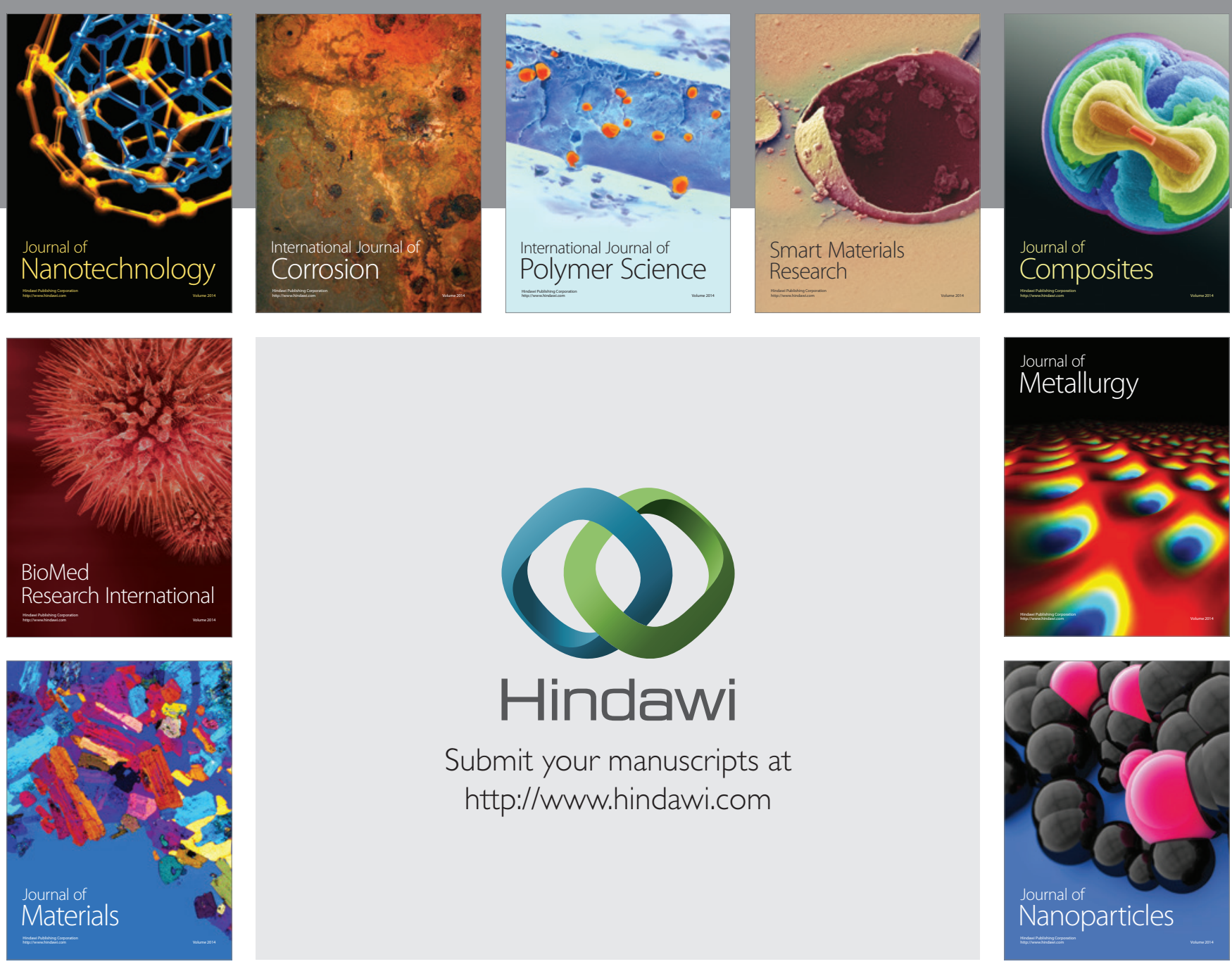

Submit your manuscripts at http://www.hindawi.com
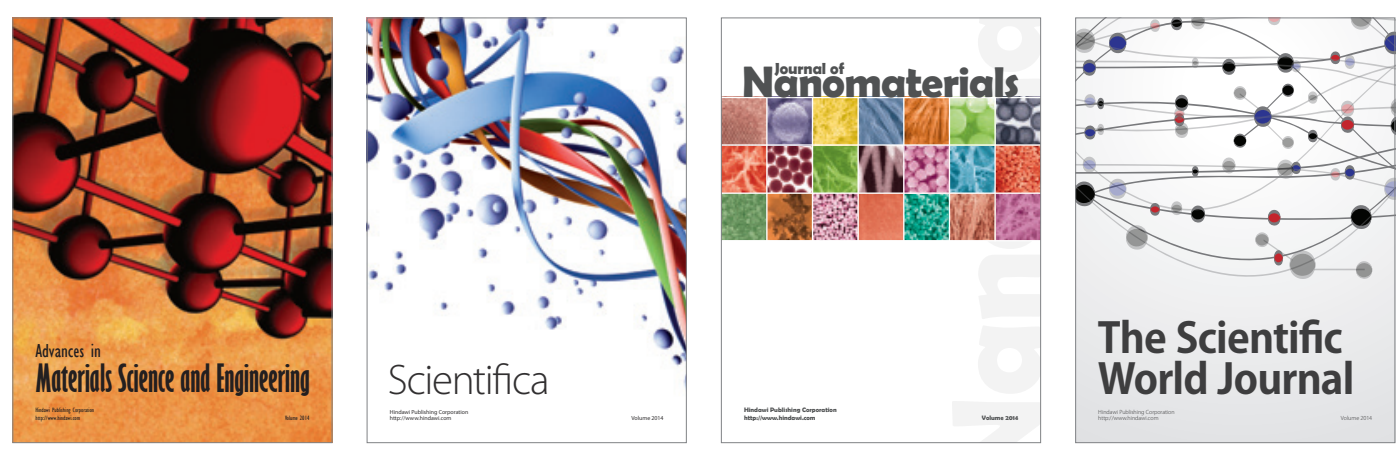

\section{The Scientific World Journal}
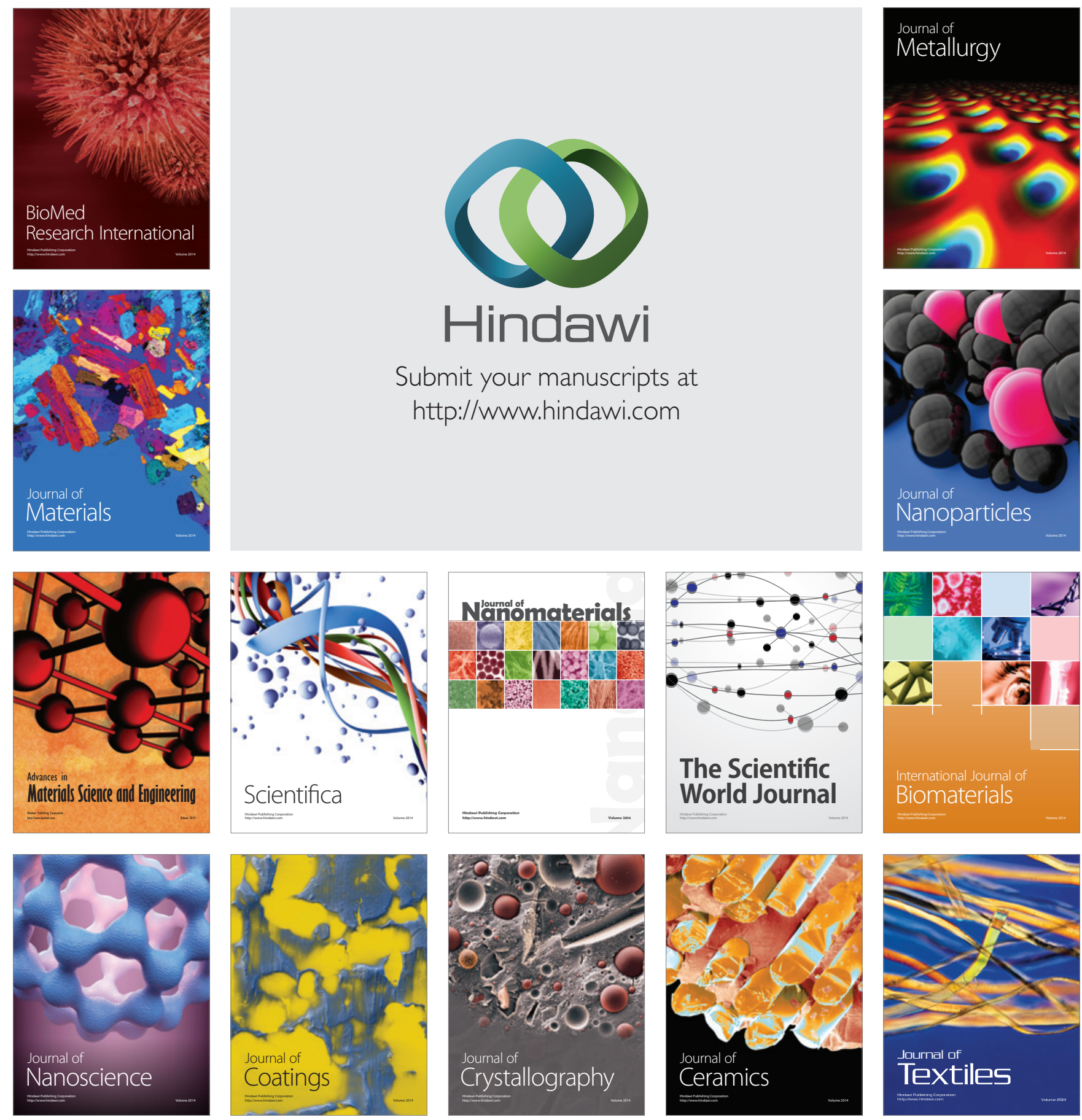